\title{
Prevalence and Characterization of Escherichia coli from Poultry Meat in Bhubaneswar
}

\author{
Iswar Anand Senapati ${ }^{1 *}$, Rajashree Mishra ${ }^{1}$, Akshaya Kumar Kundu ${ }^{2}$, \\ Bidyut Prava Mishra ${ }^{3}$ and Prasana Kumar Rath ${ }^{4}$ \\ ${ }^{1}$ Department of Veterinary Microbiology, ${ }^{2}$ Department of Veterinary Physiology, ${ }^{3}$ Department \\ of Livestock Products Technology, ${ }^{4}$ Department of Veterinary Pathology, College of \\ Veterinary Science and Animal Husbandry, OUAT, Bhubaneswar, India \\ *Corresponding author
}

\section{A B S T R A C T}

\begin{tabular}{|l|}
\hline Ke y w o r d s \\
$\begin{array}{l}\text { E. coli, Chicken } \\
\text { meat, Antibiotics, } \\
\text { Biofilm }\end{array}$ \\
\hline Article Info \\
$\begin{array}{l}\text { Accepted: } \\
17 \text { August } 2020 \\
\text { Available Online: } \\
\text { 10 September } 2020\end{array}$ \\
\hline
\end{tabular}

E. coli are normal inhabitants of the gastrointestinal tract of animals, birds and humans of which only some strains have become highly adapted to cause diarrhoea and a range of extra-intestinal diseases. In the present study, a total of 320 raw poultry meat samples were collected from retail poultry meat market of Bhubaneswar, Odisha, India. A total of $224 E$. coli isolates were obtained. The triturated samples were first inoculated on MLA and then on EMB agar, the colonies with greenish metallic sheen were considered as E. coli which was further confirmed by biochemical tests. The overall prevalence of $E$. coli observed during the present study was 70 per cent in the samples. In vitro antibiotic resistance patterns against 18 antibiotics revealed highest resistance to Oxytetracycline and highest sensitivity was exhibited to Tobramycin by all types of isolates. All the 224 E. coli isolates were subjected to biofilm production by Microtiter Plate Method and Congo Red Agar method. $68.75 \%$ of $E$. coli isolates were found to be positive for biofilm formation by both MTP method and CRA method. By MTP method $12.5 \%, 79.17 \%$ and $8.33 \%$ isolates are high, moderate and weakly positive respectively. By CRA method, $20.83 \%, 50 \%$ and $29.17 \%$ isolates are high, moderate and weakly positive respectively.

\section{Introduction}

Poultry meat is a highly digestible, tasty and low-calorie food, often recommended by nutritionists over other meats. Poultry meat is often contaminated with Escherichia coli at the time of slaughtering. E coli are normal inhabitants of the gastrointestinal tract of animals, birds and humans of which some strains have become highly adapted to cause diarrhoea and a range of extra-intestinal diseases. The organism continues to provide new challenges to food safety or public health. The work has been conducted for the prevalence and characterization of $E$. coli from broiler birds' meat in and around Bhubaneswar, Odisha. They were screened for biofilm formation and antimicrobial susceptibility. 


\section{Materials and Methods}

The present study was undertaken with a view to isolate E. coli from different samples of poultry meat sold in retail markets of Bhubaneswar and to study their properties in relation to biochemical characters, in vitro antimicrobial drug resistance pattern and biofilm forming capacity. The recovered isolates were confirmed on the basis of their morphological, cultural and biochemical characteristics. Altogether 320 samples comprising of breast muscle and leg muscle (160 samples each) were collected from randomly selected ten different poultry meat shops in Bhubaneswar, under aseptic measures. The samples were collected in sterile plastic containers and transported to the laboratory in an ice box as soon as possible. All samples were immediately marked post collection. Each sample was thoroughly triturated in a sterile mortar and pestle before inoculating on media for isolation. The samples thus collected were subjected to bacteriological examination. Isolation of $E$. coli was attempted from different samples of meat according to Edwards and Ewing (1972), Cruickshank et al., (1975) and Quinn et al., (1994) by using MacConkey lactose agar (MLA) and Eosin Methylene Blue (EMB) agar as selective media.

After receiving at laboratory the samples were unpacked aseptically. A small piece was cut out of each sample and ground using mortar and pestle with one time addition of few milliliters of normal saline. Then a $1 \mathrm{ml}$ of meat paste was inoculated with BHI broth. After an incubation of 18 hours the culture was considered for Gram Stain. Those cultures which revealed the presence of Gram Negative bacilli were passed on for further tests. Each sample having Gram Negative rods were inoculated on MLA and incubated at $37^{\circ} \mathrm{C}$ for 24 hours. The pink colonies were streaked on EMB and incubated at $37^{\circ} \mathrm{C}$ for 24 hours. Dark colonies with greenish nucleated metallic sheen after 24 hours of inoculation at $37^{\circ} \mathrm{C}$ were considered to be of typical E. coli. They were transferred to nutrient agar (NA) slants so as to sustain them for further identification and characterization. The identification of the isolates was done according to the methods described by Sojka (1965), Edwards and Ewings (1972), Cruickshank et al., (1975) and Kreig and Holt (1984). The isolates were studied for their biochemical characters viz., IMViC (Indole, Methyl Red, Voges-Proskauer and Citrate) reactions.

The antibiotic susceptibility tests were performed as per method described by Bauer et al., (1966) to find out the antibiotic resistance pattern of all E. coli isolates. In vitro antibiotic sensitivity test of the isolates was conducted by paper disc diffusion method using the disc supplied by HiMedia Laboratories Pvt. Ltd., Mumbai, India. Isolates were subjected to antimicrobial sensitivity test against 18 antibiotics such as Amikacin (AK30), AmoxyClav (AMC30), Ampicillin/Cloxacillin (AX10), Cefepime (CPM30), Ceftriaxone (CTR30), Cephotaxime (CTX30), Chloramphenicol (C30), Ciprofloxacin (CIP5), Co-Trimoxazole (COT25), Doxyxycline (DO10), Gentamicin (GEN10), Imipenem (IPM10), Nalidixic acid (NA30), Nitrofurantoin (NIT200), Norfloxacin (NX10), Oxytetracycline (O30), Streptomycin (S10) and Tobramycin (TOB10).

E. coli isolates were grown in Brain Heart Infusion (BHI) broth (HiMedia) for 12-18 hours. The grown cultures were swabbed on Mueller Hinton agar plate (HiMedia) using sterile cotton swabs and left for at least 5 minutes as pre-diffusion time. Then using an ethanol dipped and flamed forceps different antibiotic discs were placed on the agar 
surface at about two centimeters apart. The discs were slightly pressed with the forceps to make complete contact with the medium. The plates were incubated at $37^{\circ} \mathrm{C}$ for $18-24$ hours. After the incubation period, the diameter of inhibition zones were measured and compared with interpretative chart provided by the manufacturer and zones were graded as sensitive, intermediate and resistant.

Biofilm formation assay was performed by tissue culture plate method according to the protocol described by O'Toole G.A. (2011). The cultures of Escherichia coli were grown for 24 hours at $37^{\circ} \mathrm{C}$ in Tryptone Soya Broth (TSB). Cultures were added in the wells of the microtiter plate. The microtiter plate was incubated for 8 hours at $37^{\circ} \mathrm{C}$. After incubation the cells were dumped out by turning the plate over and shaking out the liquid. The plate was gently submerged in a small tub of water and the water was shaken out. The process was repeated for a second time. This step helps remove unattached cells and media components that can be stained in the next step and significantly lowers the background staining. $125 \mu \mathrm{l}$ of a $0.1 \%$ solution of crystal violet in water was added to each well of the microtiter plate. The microtiter plate was incubated at room temperature for 15 minutes. The plate was rinsed 4 times with water by submerging in a tub of water followed by shaking out and blotting vigorously on a stack of paper towel to rid the plate of all excess cells and dye. The microtiter plate was turned upside down and left for drying. For qualitative assay the wells were photographed after drying. Freeman et al., (1989) method was followed with slight modification. The Medium was composed of Tryptone soya agar (HiMedia, Mumbai) $40 \mathrm{~g} / \mathrm{l}$, sucrose $50 \mathrm{~g} / \mathrm{l}$ and Congo red $0.8 \mathrm{~g} / \mathrm{l}$. Congo red stain was prepared as a concentrated aqueous solution and autoclaved $\left(121^{\circ} \mathrm{C}\right.$ for 15 minutes) separately from the other medium constituents, and was then added when the agar had cooled to $55^{\circ} \mathrm{C}$.
Plates of the medium were inoculated and incubated aerobically for 24 hours at $37^{\circ} \mathrm{C}$. A positive result was indicated by black colonies with dry crystalline consistency. Non-slime producers usually remained pink, though occasional darkening at the centre of the colonies was observed and this gave a bulls eye appearance. An indeterminate result was indicated by a darkening of the colonies but with the absence of a dry crystalline colonial morphology. Isolates presenting two tones of black, bright black (BB) and dry opaque black (OB), were classified as negative.

\section{Results and Discussion}

Isolation and Identification of $E$. coli isolates

During present study, an attempt was made to isolate $E$. coli from the raw poultry meat samples from retail meat market of Bhubaneswar. 224 (70.00\%) E. coli isolates were obtained from 320 raw poultry meat samples. All the isolates revealed characteristic features of $E$. coli, which were Gram negative bacilli, by producing characteristic pink colonies on MLA and colonies with greenish metallic sheen on eosin methylene blue agar (EMB). On preliminary biochemical characterization they revealed characteristic IMViC pattern that is ++ - -. Almost similar result was found by Nzouankeu et al., (2010), who collected 150 poultry meat samples from eight retail markets in Yaounde. However, lower isolation rate was reported by Dutta et al., (2011) who also recovered E. coli isolates from chickens. The report of isolation of $E$. coli by various workers; Silva et al., (2011) isolated Escherichia coli from chicken livers from two slaughter houses were genotypically characterized in 62 samples. Thirty samples were macroscopically unchanged and 32 demonstrated alterations that led to the disposal of carcass for sanitary inspection. 
Thirty Escherichia coli strains from 21 unchanged and 9 from carcasses that were rejected were isolated through the classical method. Zhao et al., (2012) recovered Escherichia coli from the National Antimicrobial Resistance Monitoring System retail meat program and examined for antimicrobial susceptibility. They screened 11,921 retail meat samples from four U.S. states collected during 2002-2008 which consist of 2,988 chicken breasts. They found that 83.5 per cent chickens were contaminated with the organism.

\section{Antimicrobial drug resistance among the} $E$. coli isolates

Among 224 E. coli isolates the highest resistance was observed against the Oxytetracycline $(64.73 \%)$ followed by Chloramphenicol (58.48\%), Ampicillin/ Cloxacillin (57.14\%), Cephotaxime (54.02\%), Amoxycillin + Clavulanic acid (50.00\%), Ceftriaxone (49.55\%), Streptomycin (47.32\%), Doxyxycline (36.16\%), Amikacin (36.16\%), Co-Trimoxazole (33.93\%), Nitrofurantoin (29.46\%), Nalidixic acid (23.21\%), Gentamicin (22.78\%), Ciprofloxacin (22.32\%), Norfloxacin $(16.96 \%)$, Tobramycin $(11.16 \%)$, Imipenem $(5.80 \%)$ and least resistance was observed against Cefepime (5.36\%). Highest sensitivity was exhibited to Tobramycin (88.84\%) followed by Imipenem $(86.16 \%)$, Cefepime (85.71\%), Norfloxacin (79.02\%), Ciprofloxacin (77.68\%), Nalidixic acid (75.00\%), Nitrofurantoin (70.54\%), CoTrimoxazole $(60.71 \%)$, Gentamicin $(58.93 \%)$, Amikacin (58.48\%), Doxyxycline (52.23\%), Streptomycin (46.88\%), Amoxycillin + Clavulanic acid (48.21\%), Ceftriaxone (46.88\%), Cephotaxime (44.20\%), Chloramphenicol (40.18\%), Ampicillin/ Cloxacillin (35.71\%) and Oxytetracycline $(32.59 \%)$. This is being depicted in Table 1 and Figure 1.
In the present study highest per cent of isolates $(64.73 \%)$ were resistant to oxytetracycline. While lower resistance was reported by Singh et al., (1992) and Bogaard et al., (2001). Moderately higher resistance of $58.48 \%$ has been noticed against Chloramphenicol. Similar findings have also been reported by Cid et al., (1996). Higher resistance was reported by. While lower resistance was reported by Sharada et al., (2010), Dash et al., (2012), Zhao et al., (2012), Pavlickova et al., (2017) and Somda et al., (2018). Moderate per cent $(57.14 \%)$ of isolates were found resistant to Ampicillin/Cloxacillin. Similar findings have also been reported by Singh et al., (1992) and Somda et al., (2018). The results are in contrast to the higher resistance as reported by Cid et al., (1996), Dash et al., (2012) and Suzuki et al., (2019). Lower resistance has been reported by Aksoy et al., (2007), Zhao et al., (2012) and Pavlickova et al., (2017). In the present study moderate per cent $(54.02 \%)$ of isolates were found resistant to Cephotaxime. Lower resistance has been reported by Aksoy et al., (2007), Dash et al., (2012), Pavlickova et al., (2017), Somda et al., (2018) and Bantawa et al., (2019). $50.00 \%$ isolates were found resistant to Amoxycillin + Clavulanic acid. The result is in accordance to moderate resistance as reported by Somda et al., (2018). Bantawa et al., (2019) reported $100 \%$ resistance to amoxycillin. In contrast to the findings, lower resistance was reported by Aksoy et al., (2007), Zhao et al., (2012) and Pavlickova et al., (2017). In the study moderate per cent $(49.55 \%)$ of isolates were found resistant to Ceftriaxone. Similar findings have also been reported by Singh et al., (1992) and Somda et al., (2018). The results are in contrast to the higher resistance as reported by Cid et al., (1996), Dash et al., (2012) and Suzuki et al., (2019). 
Table.1 In vitro antimicrobial drug resistance pattern of $E$. coli isolates

\begin{tabular}{|c|c|c|c|c|}
\hline Sl. No. & Antimicrobial agents & Sensitive & Intermediate & Resistance \\
\hline 1 & Amikacin (AK) & $\begin{array}{c}131 \\
(58.48 \%)\end{array}$ & $12(5.36 \%)$ & $81(36.16 \%)$ \\
\hline 2 & $\begin{array}{c}\text { Amoxycillin + Clavulanic acid } \\
\text { (AMC) }\end{array}$ & $\begin{array}{c}108 \\
(48.21 \%)\end{array}$ & $4(1.79 \%)$ & $\begin{array}{c}112 \\
(50.00 \%)\end{array}$ \\
\hline 3 & Ampicillin/Cloxacillin (AX) & $80(35.71 \%)$ & $16(7.14 \%)$ & $\begin{array}{c}128 \\
(57.14 \%)\end{array}$ \\
\hline 4 & Cefepime (CPM) & $\begin{array}{c}192 \\
(85.71 \%)\end{array}$ & $20(8.93 \%)$ & $12(5.36 \%)$ \\
\hline 5 & Ceftriaxone (CTR) & $\begin{array}{c}105 \\
(46.88 \%)\end{array}$ & $8(3.57 \%)$ & $\begin{array}{c}111 \\
(49.55 \%)\end{array}$ \\
\hline 6 & Cephotaxime (CTX) & $99(44.20 \%)$ & $4(1.78 \%)$ & $\begin{array}{c}121 \\
(54.02 \%)\end{array}$ \\
\hline 7 & Chloramphenicol (C) & $90(40.18 \%)$ & $3(1.34 \%)$ & $\begin{array}{c}131 \\
(58.48 \%)\end{array}$ \\
\hline 8 & Ciprofloxacin (CIP) & $\begin{array}{c}174 \\
(77.68 \%)\end{array}$ & $0(0.00 \%)$ & $50(22.32 \%)$ \\
\hline 9 & Co-Trimoxazole (COT) & $\begin{array}{c}136 \\
(60.71 \%)\end{array}$ & $12(5.36 \%)$ & $76(33.93 \%)$ \\
\hline 10 & $\begin{array}{l}\text { Doxyxycline Hydrochloride } \\
\text { (DO) }\end{array}$ & $\begin{array}{c}117 \\
(52.23 \%)\end{array}$ & $26(11.61 \%)$ & $81(36.16 \%)$ \\
\hline 11 & Gentamicin (GEN) & $\begin{array}{c}132 \\
(58.93 \%)\end{array}$ & $41(18.30 \%)$ & $51(22.78 \%)$ \\
\hline 12 & Imipenem (IPM) & $\begin{array}{c}193 \\
(86.16 \%)\end{array}$ & $18(8.04 \%)$ & $13(5.80 \%)$ \\
\hline 13 & Nalidixic acid (NA) & $\begin{array}{c}168 \\
(75.00 \%)\end{array}$ & $4(1.78 \%)$ & $52(23.21 \%)$ \\
\hline 14 & Nitrofurantoin (NIT) & $\begin{array}{c}158 \\
(70.54 \%)\end{array}$ & $0(0.00 \%)$ & $66(29.46 \%)$ \\
\hline 15 & Norfloxacin (NX) & $\begin{array}{c}177 \\
(79.02 \%)\end{array}$ & $9(4.02 \%)$ & $38(16.96 \%)$ \\
\hline 16 & Oxytetracycline $(\mathrm{O})$ & $73(32.59 \%)$ & $6(2.68 \%)$ & $\begin{array}{c}145 \\
(64.73 \%)\end{array}$ \\
\hline 17 & Streptomycin (S) & $\begin{array}{c}105 \\
(46.88 \%)\end{array}$ & $13(5.80 \%)$ & $\begin{array}{c}106 \\
(47.32 \%)\end{array}$ \\
\hline 18 & Tobramycin (TOB) & $\begin{array}{c}199 \\
(88.84 \%)\end{array}$ & $0(0.00 \%)$ & $25(11.16 \%)$ \\
\hline
\end{tabular}


Figure.1 In vitro antimicrobial drug resistance pattern of $E$. coli isolates

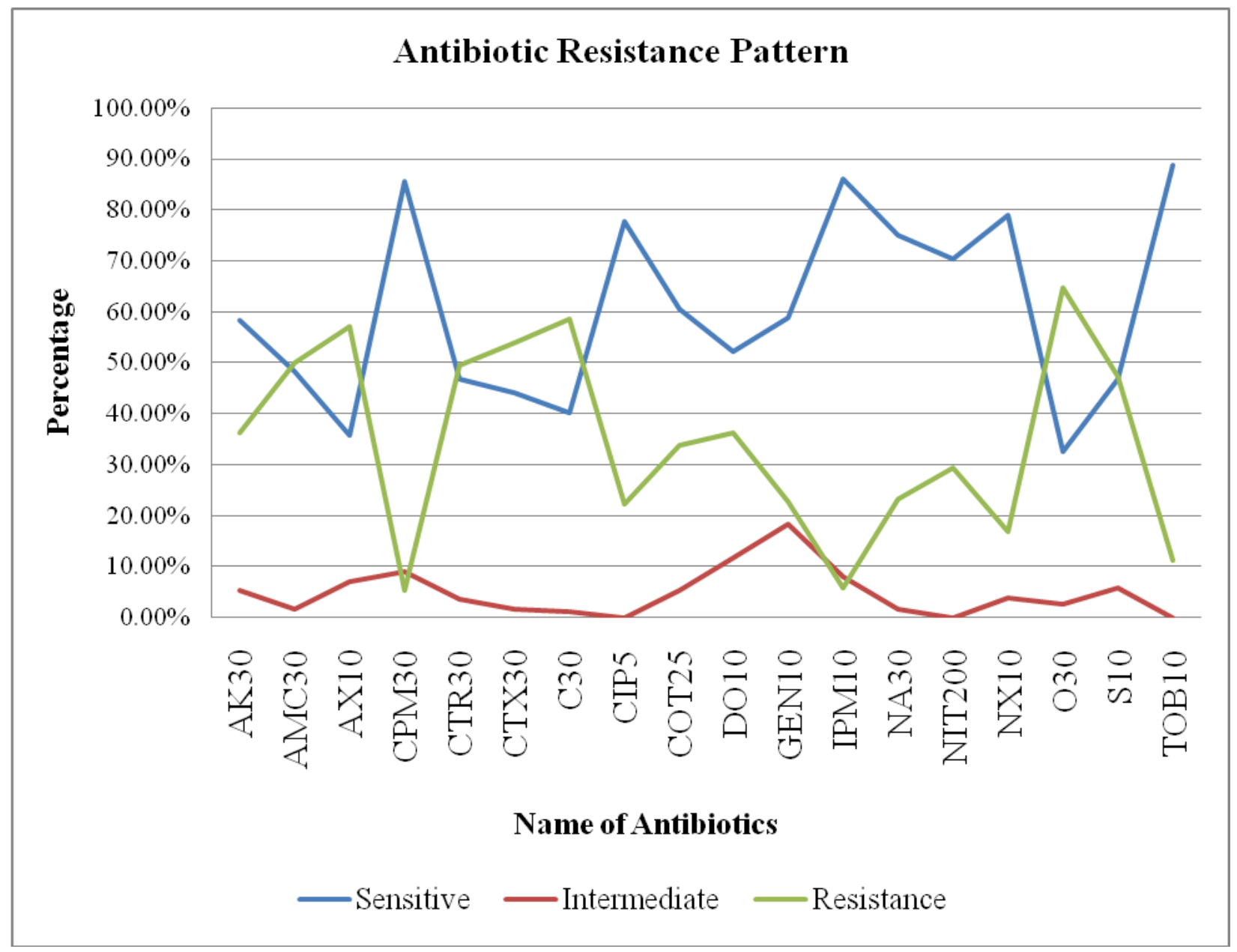

Lower resistance has been reported by Zhao et al., (2012) and Somda et al., (2018). $47.32 \%$ of isolates had shown resistance to Streptomycin in this study. Higher resistance was reported by Cid et al., (1996) and Schroeder et al., (2002). Dash et al., (2012) observed $100 \%$ resistance towards Streptomycin. Lower resistance was observed by Aksoy et al., (2007) and Zhao et al., (2012). Doxycycline was found to have resistance of $36.16 \%$ and a sensitivity of $52.23 \%$ in the present study. In contrast to those, Pavlickova et al., (2017) found less resistant and more sensitive isolates. Highest number $(88.84 \%)$ of isolates in this study are sensitive to Tobramycin. It is in agreement with Cid et al., (1996). In our study Imipenem holds the second position in terms of number of isolates being sensitive to it $(86.16 \%)$. It is in accordance with Pavlickova et al., (2017). Somda et al., (2018) reported 100\% sensitivity towards Imipenem. In present study $85.71 \%$ of isolates were found to be sensitive to Cefepime which is in agreement with Pavlickova et al., (2017). Norfloxacin received sensitivity from $79.02 \%$ isolates. It is in accordance with Sharada et al., (2010). In contrast quite lower sensitivity was obtained by Alimehr et al., (1999). For Ciprofloxacin, $77.68 \%$ sensitivity of isolates was observed in this study which is in accordance with Sharada et al., (2010), Dash et al., (2012) and Pavlickova et al., (2017). Higher sensitivity was observed by Zhao et al., (2012) and 
Somda et al., (2018). In this study $75 \%$ isolates are sensitive towards Nalidixic acid. It is in accordance with Bantawa et al., (2019). Higher values are obtained by Cid et al., (1996), Zhao et al., (2012) and Somda et al., (2018). 70.54\% isolates are sensitive to Nitrofurantoin in this study. Higher sensitivity was observed by Karlowsky et al., (2003) and Zykov et al., (2015). Co-trimoxazole had sensitivity of $60.71 \%$ isolates. It is in accordance with Cid et al., (1996) and Somda et al., (2018). Higher sensitivity was observed by Schroeder et al., (2002), Aksoy et al., (2007) and Pavlickova et al., (2017).

Lower sensitivity was reported by Sharada et al., (2010). Gentamicin was sensitive to $58.93 \%$ isolates in this study. Higher sensitivity was projected by Singh et al., (1992), Cid et al., (1996), Schroeder et al., (2002), Aksoy et al., (2007), Zhao et al., (2012) and Somda et al., (2018). In contrast to these, lower sensitivity was observed by Dash et al., (2012) and Pavlickova et al., (2017). In this study Amikacin got sensitivity from $58.48 \%$ isolates. It is in accordance with Dash et al., (2012). Higher sensitivity was reported by Cid et al., (1996).

\section{Biofilm formation assay}

There is growing appreciation that formation of bacterial surface communities is a process that contributes to pathogenicity of microorganisms (Parsek et al., 2004). It has become a common working hypothesis that the persistence of bacterial biofilms in the human body is a major cause of recurrent or chronic infections (Hall-Stoodley et al., 2005). In addition, formation of bacterial associations within bladder epithelium is characteristic of experimental urinary tract infections (UTI) (Kau et al., 2005). Many such reports are being published, from all over the world, revealing biofilm forming pathogenic $E$. coli an emerging threat to animal as well as public health. Since most of the presently available antibiotics are ineffective against such exopolysaccharide, it could lead to a crisis in public health in near future. That's why biofilm formation assay was being conducted under the present study. All the 224 E. coli isolates were subjected to biofilm production by Microtiter Plate Method and Congo Red Agar method.

In Microtiter plate method, 154 out of 224 isolates $(68.75 \%)$ were found to be positive for biofilm formation. Among those, $7.14 \%$ isolates are highly positive, $70.78 \%$ isolates are moderately positive and $27.08 \%$ are weakly positive. Also, in Congo Red Agar method, $68.75 \%$ isolates produced biofilm. $23.38 \%$ isolates are highly positive, $37.66 \%$ isolates are moderate positive and $38.96 \%$ are weakly positive.

Summary and conclusions are as follows:

The majority of market poultry meat samples were found to be contaminated or carried $E$. coli infection.

Around $70 \%$ of meat samples were carrying detectable level of $E$. coli which is quite a high rate of prevalence, which itself carries a public health concern. Antibiotic sensitivity testing of $E$. coli isolates revealed highest resistance of $64.73 \%$ to Oxytetracycline followed by Chloramphenicol (58.48\%), Ampicillin/C loxacillin (57.14\%) and so on. Highest sensitivity was recorded with Tobramycin $(88.84 \%)$ followed by Imipenem $(86.16 \%)$, Cefepime (85.71\%), Norfloxacin (79.02\%) and so on. This much of resistance indicates the indiscriminate and widespread use of these antimicrobials as feed additives or for prophylactic purpose in poultry industry, emphasizing strict and urgent need of restrictions in order to make the use of antibiotics judicious. 


\section{References}

Alimehr M, Sadeghi-Hashjin G, Pourbakhsh SA and Nofouzi K. 1999. Isolation, Identification and in vitro Susceptibility of Avian Escherichia coli to Selected Fluoroquinolones, Arch. Razi Ins, 50: 7782.

Aksoy A, Yildirim M, Kacmaz B, Apan TZ and Gocmen JS. 2007. Verotoxin Production in Strains of Escherichia coli Isolated from Cattle and Sheep, and Their Resistance to Antibiotics, Turk. J. Vet. Anim. Sci., 31(4): 225-231.

Bantawa K, Sah SN, Limbu DS, Subba P and Ghimire A. 2019. Antibiotic resistance patterns of Staphylococcus aureus, Escherichia coli, Salmonella, Shigella and Vibrio isolated from chicken, pork, buffalo and goat meat in eastern Nepal, BMC Res Notes, 12(766): 1-6.

Bauer AW, Kirby WMM, Sherris JC and Turck M. 1966. Antibiotic susceptibility testing by standard single disk method, Am. J. Clin. Pathol., 45: 493-496.

van den Bogaard AE, London N, Driessen C and Stobberingh EE. 2001. Antibiotic resistance of faecal Escherichia coli in poultry, poultry farmers and poultry slaughterers, Journal of Antimicrobial Chemotherapy, 47: 763-771.

Cid D, Blanco M, Blanco J, Quiteira R, Fuente $\mathrm{R}$ and Blanco J. 1996. Serogroups, toxins and antibiotic resistance of Escherichia coli strains isolated from diarrhoeic goat kids in Spain, Veterinary Microbiology, 53: 349354.

Cruickshank R, Duguid JP, Marmion BP and Swain RH. 1975. Medical Microbiology Vol. II: The Practice of Medical Microbiology, 12th edn. Churchill Livingstone, Edinburgh, London and New York.

Dash SK, Chakraborty SP, Mandal D and Roy S. 2012. Isolation And Characterization Of Multi Drug Resistant Uropathogenic Escherichia Coli From Urine Sample Of Urinary Tract Infected Patients.
International Journal of Life Science and Pharma Research, 2(1): 25-39.

Dutta TK, Roychoudhury P, Bandyopadhyay S, Wani SA and Hussain I. 2011. Detection \& characterization of Shiga toxin producing Escherichia coli (STEC) and enteropathogenic Escherichia coli (EPEC) in poultry birds with diarrhea, Indian J. Med. Res., 133: 541-545.

Edwards R and Ewing WN. 1972. Identification of Enterobacteriaceae. 3rd edn., Burgess Publishing Co., Minnesota.

Freeman DJ, Falkiner FR and Keane CT. 1989. New method for detecting slime production by coagulase negative staphylococci, Journal of Clinical Pathology, 42: 872-874.

Hall-Stoodley L and Stoodley P. 2005. Biofilm formation and dispersal and the transmission of human pathogens, Trends in Microbiology, 13(1): 7-10.

Karlowsky JA, Thornsberry C, Jones ME, and Sahm DF. 2003. Susceptibility of Antimicrobial-Resistant Urinary Escherichia coli Isolates to Fluoroquinolones and Nitrofurantoin, Clinical Infectious Diseases, 36:183-7.

Kau AL, Hunstad DA, and Hultgren SJ. 2005. Interaction of uropathogenic Escherichia coli with host uroepithelium, Curr. Opin. Microbiol., 8:54-59.

Krieg NR and Holt JG. 1984. Bergey's Manual of Systematic Bacteriology. Williams and Wilkins, Baltimore, London.

Nzouankeu A, Guy E, Thomas N and Marguerite N. 2010. Multiple contamination of chicken with Campylobacter, Escherichia coli and Salmonella in Yaounde, J. Infect. Dev. Ctries., 4(9): 583-586.

O’Toole GA. 2011. Microtiter Dish Biofilm Formation Assay, Journal of Visualized Experiments, 47: 1-3.

Parsek MR and Fuqua C. 2004. Biofilms 2003: emerging themes and challenges in studies of surface-associated microbial life, $J$. Bacteriol., 186: 4427-4440.

Pavlickova S, Klancnik A, Dolezalova M, Mozina SS and Holko I. 2017. Antibiotic resistance, virulence factors and biofilm 
formation ability in Escherichia coli strains isolated from chicken meat and wildlife in the Czech Republic, Journal of Environmental Science and Health, Part B, $0(0): 1-7$.

Quinn PJ, Carter ME, Markey B and Carter GR. 1994. Enterobacteriaceae. In : Clinical Veterinary Microbiology, Mosby Year Book Europe Limited, Lynton House, London, England. pp. 209-236.

Schroeder CM, Meng J, Zhao S, DebRoy C, Torcolini J, Zhao C, McDermott PF, Wagner DD, Walker RD and White DG. 2002. Antimicrobial Resistance of Escherichia coli O26, O103, O111, O128, and O145 from Animals and Humans, Emerging Infectious Diseases, 8(12): 14091414.

Sharada R, Ruban SW and Thiyageeswaran M. 2010. Isolation, characterization and antibiotic resistance pattern of Escherichia coli isolated from poultry, AmericanEurasian Journal of Scientific Research, 5(1): 18-22.

Silva, I .M. M., Evencio-Neto, J., Silva, R. M., Lucena-Silva, N., Magalhaes, J., Baliza, M. (2011). Genotypically characterization of Escherichia coli isolates from poultry. Arq. Bras. Med. Vet. Zootec., 63 (2): 333-339.

Singh M, Chaudhry MA, Yadava JNS and Sanyal SC. 1992. The spectrum of antibiotic resistance in human and veterinary isolates of Escherichia coli collected from 1984-86 in Northern India, Journal of Antimicrobial Chemotherapy, 29: 159-168.

Sojka WJ. 1965. Escherichia coli in domestic animals and poultry 1 st ed. Commonwealth Agricultural Bureaux, Farnham Royal,
Bucks, England.

Somda NS, Bonkoungou OJI, Zongo C, Kagambèga A, Bassolé IHN, Traoré $\mathrm{Y}$, Mahillon J, Scippo ML, Hounhouigan JD and Savadogo A. 2018. Safety of ready-toeat chicken in Burkina Faso: Microbiological quality, antibiotic resistance, and virulence genes in Escherichia coli isolated from chicken samples of Ouagadougou, Food Sci Nutr. 6: 1077-1084.

Sowers EG, Wells JG and Strockbine NA. 1996. Evaluation of Commercial Latex Reagents for Identification of $\mathrm{O} 157$ and $\mathrm{H} 7$ Antigens of Escherichia coli, Journal of Clinical Microbiology, 34(5): 1286-1289.

Suzuki Y, Hashimoto R, Xie H, Nishimura E, Nishiyama $M$, Nukazawa $K$ and Ishii $S$. 2019. Growth and antibiotic resistance acquisition of Escherichia coli in a river that receives treated sewage effluent, Science of the Total Environment, 690: 696-704.

Zhao S, Blickenstaff K, Bodeis-Jones S, Gaines SA, Tong E and McDermott PF. 2012. Comparison of the Prevalence and Antimicrobial Resistances of Escherichia coli Isolates from Different Retail Meats in the United States, 2002 to 2008, Applied and Environmental Microbiology, 78(6): 1701-1707.

Zykov IN, Sundsfjord A, Småbrekkec L and Samuelsen $\varnothing$. 2015. The antimicrobial activity of mecillinam, nitrofurantoin, temocillin and fosfomycin and comparative analysis of resistance patterns in a nationwide collection of ESBL-producing Escherichia coli in Norway 2010-2011, Infectious Diseases, 1-9.

\section{How to cite this article:}

Iswar Anand Senapati, Rajashree Mishra, Akshaya Kumar Kundu, Bidyut Prava Mishra and Prasana Kumar Rath. 2020. Prevalence and Characterization of Escherichia coli from Poultry Meat in Bhubaneswar. Int.J.Curr.Microbiol.App.Sci. 9(09): 2047-2055. doi: https://doi.org/10.20546/ijcmas.2020.909.255 